\title{
Fitness of the pMV158 replicon in Streptococcus pneumoniae
}

\author{
Ana María Hernández-Arriaga, Manuel Espinosa, and Gloria del \\ Solar* \\ Centro de Investigaciones Biológicas, Consejo Superior de Investigaciones \\ Científicas, Madrid, Spain
}

*Corresponding author. Address: Centro de Investigaciones Biológicas, Ramiro de Maeztu 9, 28040 Madrid, Spain.

E-mail address: gdelsolar@cib.csic.es.

Key words: Plasmid stability; Host-plasmid fitness; Genetic load 


\section{Abstract}

Promiscuous, rolling-circle replication plasmid pMV158 determines tetracycline resistance to its host and can be mobilized by conjugation. Plasmid pLS1 is a deletion derivative of pMV158 that has lost its conjugative mobilization ability. Both plasmids replicate efficiently and are stably inherited in Streptococcus pneumoniae. We have analyzed the effect of pMV158 and pLS1 carriage on the bacterial growth rate. Whereas the parental plasmid does not significantly modify the cell doubling time, pLS1 slows down the growth of the bacterial host by $8 \%-9 \%$. The bases of the differential burden caused by pMV158 and pLS1 carriage are not yet understood. The negligible cost of the pMV158 parental natural plasmid on the host might explain the prevalence of small, multicopy, rolling-circle replication plasmids, even though they lack any selectable trait.

\section{Introduction}

Fitness of a given gene or genome denotes the efficacy with which it is propagated in a particular environment. Hence, fitness of a plasmid genome is contributed to by both horizontal and vertical propagation processes involving the plasmid. Mechanisms such as conjugation, transformation and transduction allow a plasmid to be horizontally transferred to new individuals, thus increasing its presence in the bacterial population. On the other hand, plasmid stable inheritance as well as negligible plasmid burden on the host cell should account for an efficient vertical propagation of the plasmid genome within the bacterial population in the absence of selection for plasmid traits. A number of studies have shown that plasmid carriage imposes a metabolic cost (ranging from negligible to $40 \%$ ) on the bacterial host, which results in an increase of the cell doubling time (Godwin and Slater 1979; Helling, Kinney et al. 1981; Bouma and Lenski 1988; De Gelder, Ponciano et al. 2004; Watve, Dahanukar et al. 2010). 
In a mixed population consisting of plasmid-free and plasmid-harboring cells, the latter's lower growth rate gives rise to a decline of the fraction of plasmidcontaining cells over time due to the overgrowth of plasmid-free cells in the absence of selection for the plasmid (Lenski, Simpson et al. 1994; Foucault, Courvalin et al. 2009; San Millan, Garcia-Cobos et al. 2010). The burden for carrying a plasmid is assumed to increase with the copy number and the length of the plasmid owing to higher demands of synthesizing plasmid-encoded proteins (Smith and Bidochka 1998; Foucault, Courvalin et al. 2009; Meyer 2009; Watve, Dahanukar et al. 2010).

The promiscuous plasmid pMV158 was first isolated from Streptococcus agalactiae (Burdett 1980), and transferred to a battery of bacteria, including Firmicutes, Actinobacteria, and the Gammaproteobacterium Escherichia coli. Plasmid pMV158 is the prototype of a rolling-circle replication (RCR) plasmid family whose members share sequence homology as well as a similar genetic organization at the level of the replicon, which, in these plasmids, has been

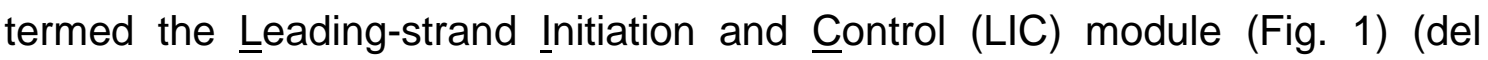
Solar, Moscoso et al. 1993). A singular feature of pMV158 is that it bears two different single strand origins (sso) for initiation of the lagging-strand synthesis, namely ssoU, which seems to be recognized in most Firmicutes, and ssoA, whose functionality appears to be restricted to streptococci (Fig. 1) (Kramer, del Solar et al. 1995; Lorenzo-Díaz and Espinosa 2009). Moreover, pMV158 contains a MOB module (Fig. 1), consisting of the oriT and the mobM gene, involved in the conjugative mobilization of the plasmid (Guzmán and Espinosa 1997; Farías and Espinosa 2000; Grohmann, Muth et al. 2003). In addition to these plasmid backbone modules that make essential contributions to the vertical and horizontal spread of the plasmid, pMV158 also harbors a constitutively expressed tetL gene encoding a membrane protein for energydependent tetracycline efflux, which confers antibiotic resistance to the bacterial host (Lacks, López et al. 1986). The pLS1 plasmid derivative was obtained in S. pneumoniae (the pneumococcus) after removal of the small EcoRI fragment of pMV158 (Fig. 1) (Stassi, López et al. 1981), and it was subsequently transferred to a variety of bacteria. Deletion in pLS1 includes $s s o U$, oriT, and part of the mobM gene. Plasmids pMV158 and pLS1 accumulate similarly low amounts of single-stranded (ss) DNA intermediates in pneumococcus, since $s s o A$, the only 
lagging-strand origin still present in pLS1, is efficiently recognized in this bacterium (Kramer, del Solar et al. 1995). In spite of having an intact LIC module, pLS1 shows a copy number lower than the parental plasmid (Kramer, del Solar et al. 1995), the reason of which remains unknown.

In the present work, we have analyzed the fitness of pMV158 and pLS1 in pneumococcus cultured in laboratory conditions by measuring the effect of the presence of either plasmid on the bacterial growth rate. We have found that, whereas the parental plasmid does not impose a measurable metabolic cost on the pneumococcal host, the deleted derivative increases slightly but significantly the cell doubling time.

\section{Materials and methods}

\subsection{Bacterial strains}

S. pneumoniae 708 (end-1 exo-1 trt-1 hex-4 malM594; (Lacks and Greenberg 1977) lacking any plasmid or harboring either pMV158 or pLS1 were used throughout the work. Pneumococcal cells were grown in $\mathrm{AGCH}$ medium (Lacks 1968 ) supplemented with $0.3 \%$ sucrose and $0.2 \%$ yeast extract, with selection for resistance to tetracycline $(\mathrm{Tc}, 0.6 \mu \mathrm{g} / \mathrm{ml})$ when required. All cultures were grown at $37^{\circ} \mathrm{C}$.

2.2. Growth kinetics of plasmid-free and pMV158- or pLS1-bearing pneumococcal cells

\subsubsection{Determination of the growth rate}

Pure cultures of plasmid-free and plasmid-containing pneumococcal strains were prepared in non-selective medium and incubated until an optical density (OD) at $650 \mathrm{~nm}$ of 0.1 was reached. This was considered as the initial $\mathrm{OD}_{650}\left(\mathrm{OD}_{0}\right.$ at $\left.t=0\right)$, above which accurate absorbance values could be obtained in the Spectronic $20_{\mathrm{D}+}$. Then, the growth rate of the pneumococcal cells was determined by measuring the $\mathrm{OD}_{650}$ of the exponentially growing cultures every $15 \mathrm{~min}$. The logarithm of the ratio between the $\mathrm{OD}_{650}$ at time $t$ 
(OD) and the $O D_{0}$ was plotted against time, and the cell doubling time ( $T$ ) was calculated from the slope $(s)$ of the following linear regression model:

$$
\log \left(\mathrm{OD} / \mathrm{OD}_{0}\right)=s t ; \quad \text { where } s=\log 2 / \mathrm{T}
$$

The kinetic analysis was performed with two independent cultures of each pneumococcal strain. The cultures were never allowed to reach the stationary phase, as these pneumococcal strains are prone to autolysis shortly after entering this phase.

\subsubsection{Competition experiments}

The ratio between the doubling times of plasmid-containing and plasmidfree pneumococcal cells was determined in competition experiments whereby the strain lacking any plasmid was co-cultured, in the absence of tetracycline, with either the pMV158- or the pLS1-containing strain. In every competition experiment, the initial fraction of plasmid-containing and plasmid-free cells was about 0.5 for each in a total bacterial population of $\sim 10^{5}$ colony-forming units $(\mathrm{CFU}) / \mathrm{ml}$. The mixed culture was successively diluted 1:1000 in pre-warmed non-selective medium when $\mathrm{OD}_{650} \sim 0.3-0.5$ was reached. In parallel, a pure culture of the corresponding plasmid-harboring strain was prepared in the same medium at an initial cell concentration of $\sim 10^{5} \mathrm{CFU} / \mathrm{ml}$. This control culture was diluted 1:1000 each time the mixed culture was, irrespective of its $O D_{650}$, which was recorded and used to calculate the number of generations occurred for the plasmid-containing cells. Before diluting the cultures, samples were withdrawn in order to determine the fraction of plasmid-harboring and plasmid-free cells by plating in solid medium that either contains or lacks tetracycline.

From competition experiments, the ratio $(\mathrm{Y})$ between the doubling times of plasmid-carrying $\left(\mathrm{T}_{+}\right)$and plasmid-free ( $\left.\mathrm{T}_{-}\right)$cells was calculated by the following mathematical approach:

we define $Y=T_{+} / \tau_{-}$. For a mixed culture that has been grown for a time $t$ in the absence of plasmid selection, the number of generations occurred for plasmidharboring $\left(\mathrm{n}_{+}\right)$and plasmid-free $\left(\mathrm{n}_{-}\right)$cells is 


$$
\mathrm{n}_{+}=t / \mathrm{T}_{+} \quad \text { and } \quad \mathrm{n}_{-}=t / \mathrm{T}_{-}=t \mathrm{Y} / \mathrm{T}_{+}=\mathrm{Y} \mathrm{n}_{+}
$$

Assuming that the plasmid is stably inherited to the daughter cells, the fraction of plasmid-harboring cells after $n_{+}$generations would be,

$$
f_{+}\left(n_{+}\right)=f_{+}(0) 2^{n_{+}} /\left(f_{+}(0) 2^{n_{+}}+f_{-}(0) 2^{\gamma n_{+}}\right)
$$

where $f_{+}(0)$ and $f_{-}(0)$ are, respectively, the initial fractions of plasmid-harboring and plasmid-free cells. The above equation was developed, assuming that $f_{+}\left(n_{+}\right)$ $+f_{-}\left(n_{+}\right)=1$, to obtain:

$$
f_{+}\left(n_{+}\right) f_{-}(0) / f_{+}(0) f_{-}\left(n_{+}\right)=2^{(1-\gamma) n_{+}}
$$

which can be converted into a linear function by taking logarithms,

$$
\log \left(f_{+}\left(n_{+}\right) f_{-}(0) / f_{+}(0) f_{-}\left(n_{+}\right)\right)=(1-\gamma) \log 2 n_{+}
$$

Experimental values of $\log \left(f_{+}\left(n_{+}\right) f_{-}(0) / f_{+}(0) f_{-}\left(n_{+}\right)\right)$were plotted against $n_{+}$, and $\mathrm{Y}$ was calculated from the slope $(s)$ of the linear regression model as $Y=1-(s /$ $\log 2)$.

From the control culture of plasmid-harboring cells, the experimental plasmid loss rate $\left(L_{e x}\right)$ was calculated from the equation:

$$
f_{+}=\left(1-L_{e x}\right)^{n_{+}} \quad(\text { Gerdes, Larsen et al. 1985; del Solar, Kramer et al. }
$$
1993),

which can be converted into a linear function by taking logarithms,

$$
\log \left(f_{+}\right)=\log \left(1-L_{e x}\right) n_{+}
$$

where log $\left(1-L_{e x}\right)$ is the slope of the linear regression fit in the plot of the experimental values of $\log \left(f_{+}\right)$against $n_{+}$. 


\section{Results and discussion}

Previous characterization of pMV158 has shown a number of features that contribute to the plasmid fitness: i) pMV158 has one of the most promiscuous replicons described so far, replicating in a variety of species, genera and phyla (del Solar, Giraldo et al. 1998); ii) pMV158 has a MOB cassette that enables it to be mobilized with the aid of functions provided by an auxiliary conjugative plasmid (Farías and Espinosa 2000); iii) the replication control system of pMV158 is properly expressed in most plasmid hosts, so that it efficiently maintains a strictly regulated, high enough copy number to allow plasmid segregational stability in the absence of any apparent active partition function (for illustrative reviews on the effect of copy number on plasmid segregational stability see (Nordström and Austin 1989; Nordström 2006).

Despite pMV158 being one of the best-known RCR plasmids, studies about whether its carriage imposes a significant burden on the bacterial hosts, hence decreasing plasmid fitness, had not been performed so far. In this work, we have started this analysis, employing the pneumococcal host, which we have been working with for the last 25 years (del Solar, Puyet et al. 1987). Although pMV158 is small $(5.5 \mathrm{~kb})$, its medium-high copy number ( 36 per chromosome equivalent) in S. pneumoniae (Lacks, López et al. 1986; Kramer, del Solar et al. 1995) and constitutively expressed tetL gene are expected to increase the metabolic burden on the host (Björkman and Andersson 2000; Foucault, Courvalin et al. 2009; Meyer 2009; Foucault, Depardieu et al. 2010). We have also analyzed the pMV158-derivative pLS1 (4.4 kb), which harbors an intact wild-type LIC module and exhibits a lower copy number ( 22 per chromosome equivalent) than the parental plasmid in the pneumococcal host (Kramer, del Solar et al. 1995). Both pMV158 and pLS1 contain lagging-strand origins efficiently recognized in this bacterium, namely $s s o U$ and $S S O A$ in pMV158 or only $s S O A$ in pLS1, and therefore very small amounts of SsDNA replication intermediates are accumulated in the cells hosting either plasmid (Kramer, del Solar et al. 1995). Both plasmids are also stably inherited in the pneumococcal host (del Solar, Kramer et al. 1993). 
In order to analyze the cost for carrying pMV158 or pLS1, the exponential growth rates of the pneumococcal cells lacking any plasmid or harboring either plasmid were determined. The growth kinetics of two independent cultures of each strain, growing in non-selective medium from an $\mathrm{OD}_{0} \sim 0.1$ to the beginning of the stationary phase, are displayed in Figure 2. From the experimental data, mean doubling times $(\mathrm{T}$ ) of $30.1 \mathrm{~min}, 28.5 \mathrm{~min}$ and $34.4 \mathrm{~min}$ were calculated, according to equation 1, for the plasmid-free cells (Fig. 2A), and for the pMV158- (Fig. 2B) and pLS1- (Fig. 2C) harboring cells, respectively. With these values, the $\mathrm{y}$ ratio between the $\mathrm{T}$ of pMV158-containing cells to plasmid-free cells was $0.947 \pm 0.085$, and that of pLS1-containing cells to plasmid-free cells was $1.142 \pm 0.046$. When different stocks of the culture medium were used in these experiments, different $\mathrm{T}$ values, but similar $\mathrm{y}$ ratios, were obtained (not shown). These results suggest that the presence of pLS1, but not pMV158, could slow down by $10 \%-20 \%$ the growth of the pneumococcal cells in the exponential phase. However, inferences derived from this analysis could be flawed due to sampling errors arising from differences in, for instance, the cleanness of the tubes where the bacterial cells were grown, or the location of the culture tube within the waterbath/incubator (laboratory conditions in which $S$. pneumoniae is grown). The inaccuracy inherent to the determination of growth rates of pure populations can be overcome in competition experiments that compare the bacterial fitness of a mixed population, where the competing cells are present in the same environment (Lenski 1991).

Competition experiments were performed by growing, for a number of generations in non-selective medium, a mixed population consisting of the plasmid-free strain and either the pMV158- or the pLS1-carrying strain (Fig. 3). In parallel, experiments of plasmid segregational stability were carried out by growing, in the same non-selective medium and for the same number of generations, pure populations of pMV158- or pLS1-containing cells (Fig. 3). These control cultures served to confirm that, under the selected experimental conditions, these two plasmids were stably inherited by daughter cells. In fact, a meaningless and statistically insignificant $L_{e x}$ of $-0.00152 \pm 0.0018$ and an almost negligible $L_{e x}$ of $0.00290 \pm 0.00080$ per cell per generation were calculated for pMV158 and pLS1, respectively, according to equation 5 . It is worth noting that the calculated pLS1 $\mathrm{L}_{\mathrm{ex}}$ is most likely an overestimation of the 
actual value, as equation 5 does not take into account the decline in the fraction of plasmid-containing cells due to overgrowth of plasmid-free cells (see below). On the other hand, competition experiments showed that the pneumococcal strain harboring pMV158 competed well with the plasmid-free isogenic strain, so that the fraction of plasmid-containing cells was maintained during growth over several generations (Fig. 3A). From these experiments, a $y$ ratio between the doubling times of pMV158-containing and plasmid-free cells of $0.9957 \pm 0.0050$, which is not significantly different from 1 , was calculated using equation 4 . Thus, carriage of pMV158 does not impose a measurable cost on the growth rate of the pneumococcal host. In contrast, bacterial cells carrying pLS1 were overgrown by plasmid-free cells, as shown by the decline of the fraction of pLS1-harboring cells over the generations (Fig. 3B). In this case, the calculated value of $Y=1.0857 \pm 0.0033$ shows that carriage of pLS1 has a small but significant cost on the growth rate of the host cell, which increases its doubling time by $8 \%-9 \%$.

The above results were unexpected, as the measurable burden on the host was caused by the shorter and lower copy-number of the two plasmids having the same pMV158 replicon. At present, the reasons that render pLS1 a burden to its host remain unknown. They might be related to the disturbance that also causes a decrease of the plasmid copy number relative to that of parent, pMV158. However, we cannot rule out that the genotype of the strain harboring pMV158, but not that containing pLS1, has evolved to overcome the burden of plasmid carriage during propagation for many generations under laboratory conditions with selective pressure for plasmid retention, as it has been previously reported for $E$. coli containing pACYC184 (Lenski, Simpson et al. 1994).

Although very preliminary, the present work is relevant in that it constitutes the first study on the effect of the carriage of small multicopy RCR plasmids on the growth rate of the host. The results might explain the wide natural representation of this kind of plasmids, even in the absence of selectable plasmid traits. Thus, by bearing a negligible metabolic burden due to plasmid carriage, the plasmid-containing cells could avoid outcompetition by plasmid-free cells. 


\section{Conclusions}

The promiscuous RCR plasmid pMV158 does not imposes, at least under laboratory conditions, a significant cost to $S$. pneumoniae, a bacterium closely related to its natural host. In contrast pLS1, a deleted, lower copy number but still segregationally stable pMV158-derivative, represents a burden that slows down the host cell growth by $8 \%-9 \%$. This is the first report on the metabolic burden imposed by carriage of a RCR replicon.

\section{Acknowledgments}

We thank J.A. Ruiz-Masó for critical reading of the manuscript, and M. Sanz for her help in culturing the pneumococcal cells. This study was supported by the Spanish Ministerio de Ciencia e Innovación (Grants CSD2008-00013, INTERMODS to ME; and BFU2010-19597 to GdS).

\section{References}

Björkman, J. and D. I. Andersson (2000). "The cost of antibiotic resistance from a bacterial perspective." Drug Resist. Updat. 3: 237-245.

Bouma, J. E. and R. E. Lenski (1988). "Evolution of a bacteria/plasmid association." Nature 335: 351-352.

Burdett, V. (1980). "Identification of tetracycline-resistant R-plasmids in Streptococcus agalactiae (groupB)." Antimicrobial Agents Chemotherapy 18: 753-760.

De Gelder, L., J. M. Ponciano, et al. (2004). "Combining mathematical models and statistical methods to understand and predict the dynamics of antibiotic-sensitive mutants in a population of resistant bacteria during experimental evolution." Genetics 168: 1131-1144.

del Solar, G., R. Giraldo, et al. (1998). "Replication and control of circular bacterial plasmids." Microbiol. Mol. Biol. Rev. 62: 434-464.

del Solar, G., M. G. Kramer, et al. (1993). "Replication of the promiscuous plasmid pLS1: a region encompassing the minus origin of replication is associated with stable plasmid inheritance." Mol Gen Genet. 241:97-105.

del Solar, G., M. Moscoso, et al. (1993). "Rolling circle-replicating plasmids from Gram-positive and Gram-negative bacteria: a wall falls." Mol. Microbiol. 8: 789-796.

del Solar, G., A. Puyet, et al. (1987). "Initiation signals for the conversion of single stranded to double stranded DNA forms in the streptococcal plasmid pLS1." Nucleic Acids Res. 15: 5561-5580. 
Farías, M. E. and M. Espinosa (2000). "Conjugal transfer of plasmid pMV158: uncoupling of the pMV158 origin of transfer from the mobilization gene mobM, and modulation of pMV158 transfer in Escherichia coli mediated by IncP plasmids." Microbiology 146: 2259-2265.

Foucault, M.-L., P. Courvalin, et al. (2009). "Fitness cost of VanA-type vancomycin resistance in methicillin-resistant Staphylococcus aureus." Antimicrobial Agents Chemotherapy 53(6): 2354-2359.

Foucault, M.-L., F. Depardieu, et al. (2010). "Inducible expression eliminates the fitness cost of vancomycin resistance in enterococci." Proc. Natl. Acad. Sci. USA 107: 16964-16969.

Gerdes, K., J. E. L. Larsen, et al. (1985). "Stable inheritance of plasmid R1 requires two different loci." J. Bacteriol. 161: 292-298.

Godwin, D. and J. H. Slater (1979). "The influence of the growth environment on the stability of a drug resistance plasmid in Escherichia coli K12." J. Gen. Microbiol. 111: 201-210.

Grohmann, E., G. Muth, et al. (2003). "Conjugative plasmid transfer in Grampositive bacteria." Microbiol. Mol. Biol. Rev. 67: 277-301.

Guzmán, L. and M. Espinosa (1997). "The mobilization protein, MobM, of the streptococcal plasmid pMV158 specifically cleaves supercoiled DNA at the plasmid oriT." J.Mol. Biol. 266: 688-702.

Helling, R. B., T. Kinney, et al. (1981). "The maintenance of plasmid-containing organisms in populations of Escherichia coli." J. Gen. Microbiol. 123: 129-141.

Kramer, M. G., G. del Solar, et al. (1995). "Lagging-strand origins of the promiscuous plasmid pMV158: physical and functional characterization." Microbiology 141: 655-662.

Lacks, S. A. (1968). "Genetic regulation of maltosaccharide utilization in pneumococcus." Genetics 60: 685-706.

Lacks, S. A. and B. Greenberg (1977). "Complementary specificity of restriction endonucleases of Diplococcus pneumoniae with respect to DNA methylation." J. Mol. Biol. 114: 153-168.

Lacks, S. A., P. López, et al. (1986). "Identification and analysis of genes for tetracycline resistance and replication functions in the broad-host-range plasmid pLS1." J. Mol. Biol. 192: 753-765.

Lenski, R. E. (1991). Quantifying fitness and gene stability in microorganisms. Assessing ecological risks of biotechnology. L. R. Ginzburg. Boston, Butterworth-Heinemann: 173-192.

Lenski, R. E., S. Simpson, et al. (1994). "Genetic analysis of a plasmidencoded, host genotype-specific enhancement of bacterial fitness." $\underline{\mathrm{J}}$ Bacteriol. 176(11): 3140-3147.

Lorenzo-Díaz, F. and M. Espinosa (2009). "Lagging-strand DNA replication origins are required for conjugal transfer of the promiscuous plasmid pMV158." J. Bacteriol. 191(3): 720-727.

Meyer, R. (2009). "Replication and conjugative mobilization of Broad Hostrange IncQ plasmids." Plasmid 62(2): 57-70.

Nordström, K. (2006). "Plasmid R1-Replication and its control." Plasmid 55: 126.

Nordström, K. and S. Austin (1989). "Mechanisms that contribute to the stable segregation of plasmids." Annu. Rev. Genet. 23: 37-69. 
San Millan, A., S. Garcia-Cobos, et al. (2010). "Haemophilus influenzae clinical isolates with plasmid $\mathrm{pB} 1000$ bearing bla $\mathrm{ROB}_{-1}$ : fitness cost and interspecies dissemination." Antimicrobial Agents Chemotherapy 54(4): 1506-1511.

Smith, M. A. and M. J. Bidochka (1998). "Bacterial fitness and plasmid loss: importance of culture conditions and plasmid size." Canadian J. Microbiol. 44: 351-355.

Stassi, D. L., P. López, et al. (1981). "Cloning of chromosomal genes in Streptococcus pneumoniae." Proc. Natl. Acad. Sci. USA 78: 7028-7032.

Watve, M. M., N. Dahanukar, et al. (2010). "Sociobiological control of plasmid copy number in bacteria." PLoS ONE 5: e9328

\section{Figure legends}

Fig. 1. Modular organization of pMV158. Plasmid pMV158 consists of five modules (shaded boxes): LIC, leading-strand initiation and control region, which includes the double strand origin (dso), and the genes encoding the initiator RepB protein and the two elements involved in replication control (the CopG protein and antisense RNAII); DET, tetracycline resistance determinant; MOB, conjugative mobilization region (including the oriT and the gene encoding the MobM protein); $s S O A$ and ssoU, lagging-strand initiation regions (single strand origins). The region deleted in pLS1 is indicated by the dashed line.

Fig. 2. Growth kinetics of $S$. pneumoniae plasmid-free cells (A), and pMV158(B) or pLS1- (C) harboring cells. Growth rate of each pneumococcal strain was calculated from two independent cultures (filled and open circles). The doubling times were calculated from the slope of the linear regression model according to equation 1.

Fig. 3. Competition assays between plasmid-free and either pMV158- (A) or pLS1- (B) harboring cells. Mixed cultures of plasmid-free and plasmid-harboring cells were grown exponentially in non selective medium for a number of generations. The fraction of plasmid-free and plasmid-harboring cells was determined every ten generations of the plasmid-containing cells.

The ratio between doubling times of plasmid-containing and plasmid-free cells was calculated from the slope of the linear regression model of the plotted equation 4 (black circles and lines). A pure culture of plasmid-harboring cells was used as control. The fraction of plasmid containing cells was determined every ten generations. The experimental loss rate $\left(\mathrm{L}_{e x}\right)$ was calculated from the slope of the linear regression model of the plotted equation 5 (red circles and lines). 
Figure 1

pMV158 (5540 bp)

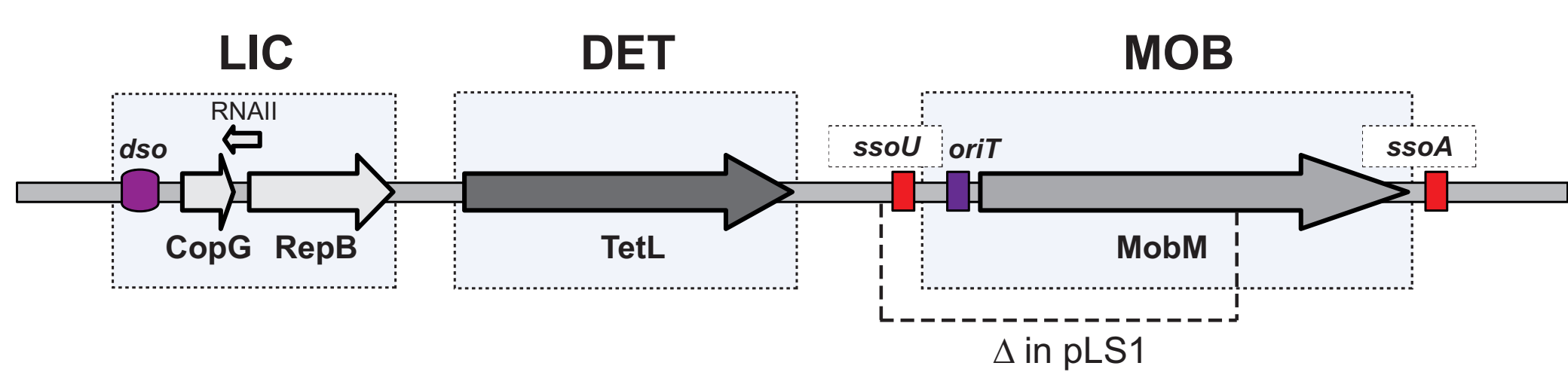

$\Delta$ in pLS1

Figure 1 


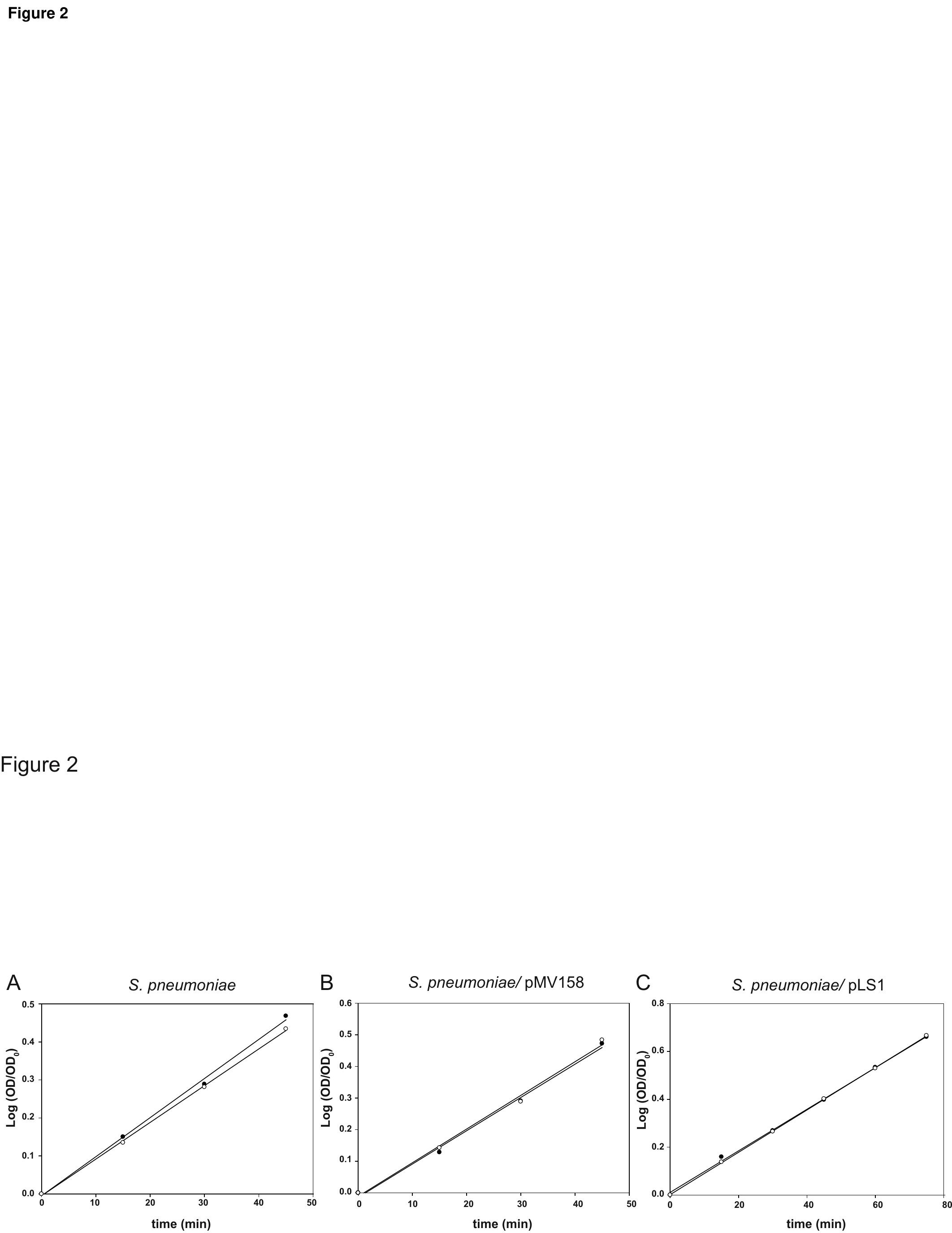

Figure 2

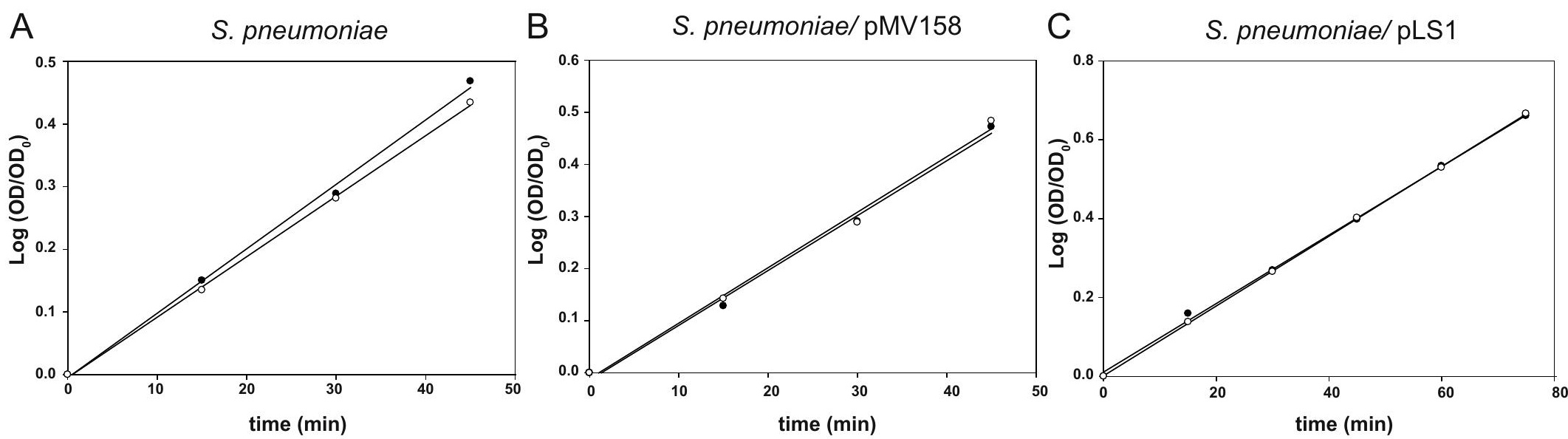$$
\text { (1.i.) }
$$ 

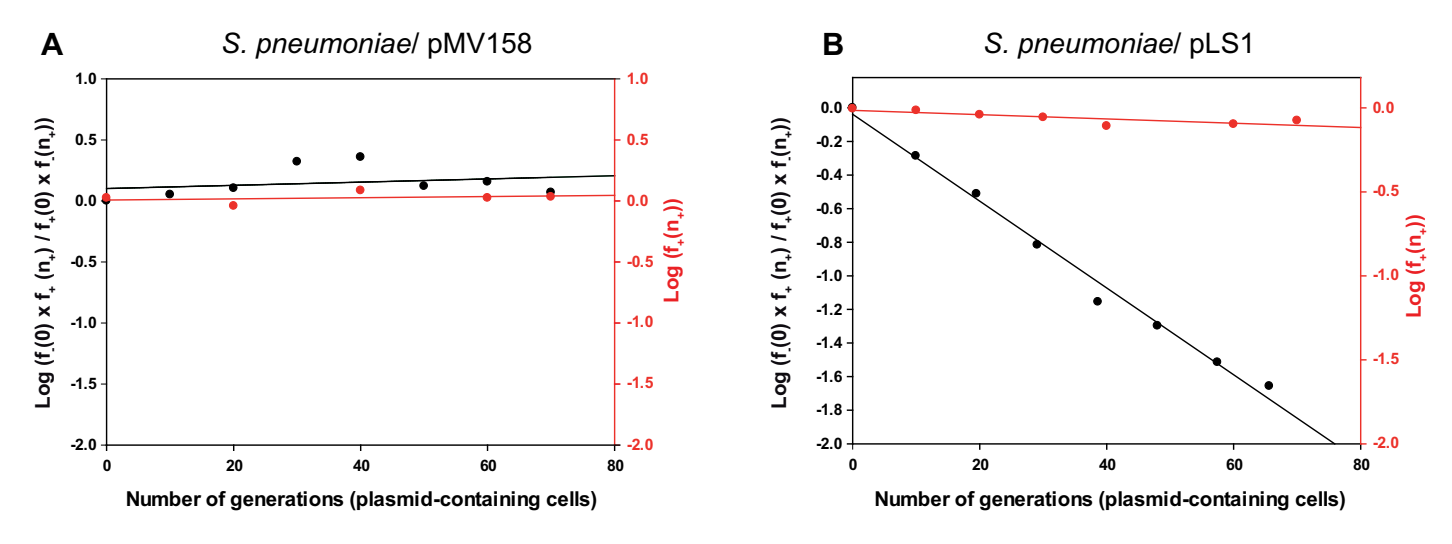

Figure 3

\section{Figure 3 \\ Figure 3}

Number of generations (plasmid-containing cells)

Number of generations (plasmid-containing cells)

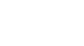

Figure 3

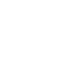
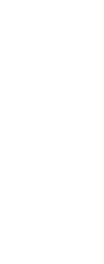

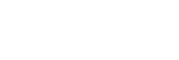

(a) 